\title{
Age-stratified outcomes after robotic-assisted laparoscopic radical prostatectomy
}

\author{
Kevin C. Zorn · Frederick P. Mendiola • \\ David E. Rapp · Albert A. Mikhail · Shang Lin · \\ Marcelo A. Orvieto - Gregory P. Zagaja • \\ Arieh L. Shalhav
}

Received: 23 October 2006/ Accepted: 8 January 2007/ Published online: 24 January 2007

(C) Springer London 2007

\begin{abstract}
We sought to evaluate post-operative return of urinary and sexual function in men undergoing robotic-assisted laparoscopic radical prostatectomy (RLRP). Prospective assessment of urinary continence and sexual function was performed in patients undergoing RLRP. Subjective assessment involved the use of the validated RAND-36 Item Health Survey/ UCLA Prostate Cancer Index questionnaire. Questionnaires were completed pre-operatively and at 1,3, 6 and 12 months post-operatively. Subset analyses were performed to assess the effect of age on functional outcomes. A total of 338 consecutive patients underwent RLRP between February 2003 and August 2005. Included patients for evaluation comprised of 21, 129, and 150 patients, aged <50, 50-59, and $\geq 60$ years old, respectively. Kaplan-Meier curve analysis demonstrated that younger men ( $<60$ years) achieved subjective continence significantly earlier than older age group ( $\geq 60$ years) $(P=0.02)$. Continence rates, however, equalized among all age groups at 1 year follow-up. Younger men $(<50$ years $)$ also demonstrated a quicker and greater return of sexual
\end{abstract}

K. C. Zorn $(\varangle)$ · F. P. Mendiola · D. E. Rapp

A. A. Mikhail - M. A. Orvieto - G. P. Zagaja .

A. L. Shalhav

Section of Urology, Department of Surgery,

University of Chicago,

5841 S. Maryland Ave. MC6038 J-653,

Chicago, IL 60637, USA

e-mail: kevinzorn@hotmail.com

S. Lin

Department of Health Studies/Statistics,

University of Chicago, Chicago, IL, USA function $(P=0.01)$, which persisted through assessment at 1 year post-operatively. Our results suggest that younger men may have an earlier return of continence and potency when compared to men > 60 years. Despite this finding, continence outcomes appear to be equal among age groups after 1 year of follow-up. Moreover, men $<60$ years continue to report superior potency outcomes compared to men $>60$ years at 1 year post-operatively. Such findings are valuable in counseling patients undergoing RLRP.

Keywords Robotic prostatectomy · Potency · Continence $\cdot$ Quality of life

\section{Introduction}

With the widespread use of PSA screening, more men are being diagnosed with prostate cancer (pCA) at an earlier age. Several open retropubic (RRP) and laparoscopic radical prostatectomy (LRP) series have suggested that younger men have better functional outcomes (continence and potency) after surgery compared to older men [1-4]. Similar data have not yet been reported for robotic-assisted laparoscopic radical prostatectomy (RLRP), despite excellent overall functional outcomes being reported in large series [5-8]. Mid-term analyses of LRP and RLRP experiences continue to show comparable oncologic results to open RRP [9]. Over the last decade, much attention has been given to health-related quality of life (HRQOL) outcomes after radical prostatectomy. In this study, we evaluate the influence of age on return of continence and potency after RLRP. 


\section{Materials and methods}

\section{Patient selection}

Between February 2003 and August 2005, 338 consecutive patients underwent RLRP for localized pCA at our institution. Of these men, two surgeries were aborted secondary to positive lymph nodes on frozen section. Eight cases were electively converted to an open technique: five due to failure to progress due to dense adhesions and poor tissue planes, one due to slow, persistent bleeding, one due to posterior bladder perforation and one due to an incidental urothelial tumor at the bladder neck. All conversions occurred during the initial 40 cases. All patients who underwent sural nerve grafting (28) were also excluded from evaluation. Patients with non-nerve sparing procedure, or those who were pre-operatively impotent, defined as having a SHIM score $<20$, were specifically excluded from sexual function analysis. One patient who was incontinent preoperatively was excluded from urinary function analysis.

\section{Surgical technique}

All RLRP cases were performed by two surgeons (A.L.S. and G.P.Z.) using the 3-arm Da Vinci Robotic System (Intuitive Surgical, Sunnyvale, CA) using our previously described technique [10-13]. Pelvic lymphadenectomy was routinely performed on men with a PSA $\geq 10 \mathrm{ng} / \mathrm{ml}$, a primary Gleason grade of 4 , or clinical stage $\geq \mathrm{T} 2 \mathrm{~b}$. All cases were approached transperitoneally with initial dissection of the seminal vesicles. The prostate was then exposed and dissected in an antegrade fashion after bladder neck transection. Nerve-sparing, when appropriate, was performed using a clipless, interfascial technique without the use of monopolar cautery. A running vesico-urethral anastomosis (VUA) was performed [14] using LapraTy clips to ensure water-tight closure [15].

Pathological analysis

All specimens were analyzed by the same uro-pathology service at our institution as described previously [16]. Positive surgical margin (PSM) was defined as tumor present at the inked margin.

\section{Functional outcome}

Functional outcomes were assessed subjectively by patient interview, as well as the validated RAND-36 Item Health Survey v2/UCLA Prostate Cancer Index
(SF-36 v2/UCLA PCI) questionnaire [17]. These HRQOL questionnaires are a self-administered, multi-item, disease-specific instrument to capture health concerns central to the quality of life of men treated for early stage prostate cancer [18]. Subjective assessment was evaluated pre-operatively, and at $1,3,6$, and 12 months post-operatively. Questionnaire administration was performed during clinic visits or via mail. Time to strict continence and potency were obtained by calculating the number of days from surgery the patient achieved urinary control and sexual function. For the purpose of outcome assessment, strict continence was defined as zero pad usage per day as well as those with occasional security pad for high levels of physical activity. Strict potency was defined as the ability to achieve penetration and complete intercourse with or without the use of oral type 5 phosphodiesterase (PDE-5) inhibitors. Patients without preoperative HRQOL surveys (10) or those with incomplete follow-up surveys were excluded from functional outcomes analysis. In addition to overall potency and continence rates, mean percent return of baseline (pROB) function was calculated at each assessment time point. The pROB was calculated as the percentage of preoperative urinary and sexual function score at all time points for each patient.

\section{Statistical analysis}

Data collection and statistical analysis was performed by a blinded third party. Continuous variables were compared using $t$-test or ANOVA while categorical variables were analyzed using the Chi-square and Fisher's exact test. Kaplan-Meier curves were constructed for various age groups to estimate the time to recovery of continence and potency. Distribution among age groups was compared using the log-rank test. Finally, subset analysis of three age groups $(<50$, $50-59$, and $\geq 60$ years old) was performed to compare functional outcomes and clinical/pathological data. A $P$-value $<0.05$ was considered statistically significant.

\section{Results}

After patient exclusion, a total of 300 consecutive men undergoing RLRP for clinically localized pCA were divided into three age groups $(<50,50-59$, and $\geq 60$ years old) comprised of 21, 129 and 150 patients, respectively. Mean overall age was 59.4 years (range 42-76). Clinical and pathologic data are summarized in Table 1. At a mean follow-up of 20.3 months, 
Table 1 Clinical and pathologic features of 300 men undergoing roboticassisted laparoscopic radical prostatectomy (RLRP) for clinically localized prostate cancer

\begin{tabular}{|c|c|c|c|c|}
\hline Variable & $\begin{array}{l}\text { Age }<50 \\
(n=21)\end{array}$ & $\begin{array}{l}\text { Age } 50-59 \\
(n=129)\end{array}$ & $\begin{array}{l}\text { Age } \geq 60 \\
(n=150)\end{array}$ & $P$-value \\
\hline $\begin{array}{l}\text { Mean age (years) } \\
\text { Pre-op potency }(\%)\end{array}$ & $46.9(42-49)$ & $55.3(50-59)$ & $64.7(60-76)$ & $<0.001$ \\
\hline Potent $($ SHIM $\geq 20)$ & $20(95.2)$ & $114(88.4)$ & $128(78.7)$ & 0.04 \\
\hline Impotent $($ SHIM $<20)$ & $1(4.8)$ & 15 (11.6) & $32(21.3)$ & \\
\hline PSA (ng/dl) & $5.2(0.6-17)$ & $6.1(0.9-25.4)$ & $6.9(1.4-32)$ & 0.09 \\
\hline \multicolumn{5}{|l|}{ Biopsy Gleason score (\%) } \\
\hline $5-6$ & $20(95)$ & $101(78)$ & $107(71)$ & 0.01 \\
\hline 7 & $0(0)$ & $27(21)$ & $38(25)$ & \\
\hline $8-10$ & $1(5)$ & $1(1)$ & $5(3)$ & \\
\hline \multicolumn{5}{|l|}{ Clinical stage $(\%)$} \\
\hline T1c & $12(57)$ & $102(79)$ & 117 (78) & 0.21 \\
\hline $\mathrm{T} 2 \mathrm{a}$ & $7(33)$ & $20(16)$ & $26(17)$ & \\
\hline $\mathrm{T} 2 \mathrm{~b}$ & $2(10)$ & $7(5)$ & $7(5)$ & \\
\hline \multicolumn{5}{|l|}{ Nerve sparing (\%) } \\
\hline Bilateral & $14(67)$ & $87(68)$ & $83(56)$ & 0.08 \\
\hline Unilateral & $7(33)$ & $30(23)$ & $43(29)$ & \\
\hline None & $0(0)$ & $11(9)$ & $23(15)$ & \\
\hline \multicolumn{5}{|c|}{ Pathology Gleason score (\%) } \\
\hline $5-6$ & $18(86)$ & $90(70)$ & $106(71)$ & 0.41 \\
\hline 7 & $2(10)$ & $34(26)$ & $39(26)$ & \\
\hline $8-10$ & $1(5)$ & $5(4)$ & $4(3)$ & \\
\hline Prostate weight $(\mathrm{g})$ & 43.3 & 47.5 & 57.2 & $<0.001$ \\
\hline & $(25.7-99.8)$ & $(10-103.1)$ & $(5.5-176)$ & \\
\hline \multicolumn{5}{|l|}{ Pathologic stage $(\%)$} \\
\hline pT2 & $16(76)$ & $111(86)$ & $124(83)$ & 0.53 \\
\hline pT3a & $5(24)$ & $15(12)$ & $20(13)$ & \\
\hline pT3b & $0(0)$ & $3(2)$ & $5(3)$ & \\
\hline \multicolumn{5}{|l|}{ Positive margins $(\%)$} \\
\hline Overall & $7(34)$ & $31(24)$ & $25(17)$ & 0.10 \\
\hline pT2 & $4(25)$ & $22(19)$ & $11(8.9)$ & 0.03 \\
\hline pT3 & $3(60)$ & $9(50)$ & $14(56)$ & 0.89 \\
\hline
\end{tabular}

93.7\% (231/300) had an undetectable PSA (less than $0.1 \mathrm{ng} / \mathrm{ml})$.

Age-stratified comparison of clinical and pathological data was performed. As expected, the prostate weights in the youngest age group was significantly smaller compared with the older patients $(P<0.01)$. Biopsy Gleason scores were significantly lower in the youngest age group $(P=0.01)$, while the differences in clinical and pathologic stages, as well as the Gleason score on final pathology were comparable among the groups. There was a trend towards higher PSMS in the youngest age group (34\%), compared with $24 \%$ and $17 \%$ in the $50-59$ and $\geq 60$ years groups, respectively, although this difference did not reach statistical significance $(P=0.10)$. The incidence of select medical co-morbidities and risk factors was assessed (diabetes, hypertension, cardiovascular diseases and smoking) and was comparable among the different age groups.
Continence outcomes

Continence outcomes of the age-stratified groups are summarized in Table 2. Patient interview revealed that 67,52 and $41 \%$ of men $<50,50-59$, and $\geq 60$ years old, respectively, achieved strict continence at 3 months. This difference was found to be statistically significant $(P=0.04)$. No statistically significant difference was seen between age groups at all other time points. Furthermore, 12-month pad-free continence rates were 86,85 and $82 \%$ for men aged $<50,50-59$ and $\geq 60$, respectively. These results are comparable among all age groups $(P=0.75)$.

Kaplan-Meier curve estimates demonstrated no significant difference in continence outcomes between the three age groups. However, comparison of men aged $<60$ and $\geq 60$ years demonstrated that younger patients achieved continence earlier than the older cohort $(P=0.02)$ (Fig. 1). 
Table 2 Clinical assessment of continence and potency after RLRP for clinically localized prostate cancer patients a Includes only pre-operative potent men with a SHIM score $\geq 20$

\begin{tabular}{lcccc}
\hline Variable & Age $<50$ & Age 50-59 & Age $\geq 60$ & $P$-value \\
\hline Continence & & & & \\
0 Pads per day $\%$ continent & & & \\
1 month & $26 \%(5 / 19)$ & $26 \%(30 / 117)$ & $17 \%(22 / 137)$ & 0.14 \\
3 months & $67 \%(12 / 18)$ & $52 \%(63 / 121)$ & $41 \%(57 / 140)$ & 0.04 \\
6 months & $82 \%(14 / 18)$ & $75 \%(91 / 122)$ & $68 \%(87 / 128)$ & 0.43 \\
12 months & $86 \%(18 / 21)$ & $85 \%(95 / 112)$ & $82 \%(106 / 130)$ & 0.75 \\
Potency & & & & \\
Bilateral NS & & & & \\
1 month & $46 \%(6 / 13)$ & $41 \%(34 / 82)$ & $36 \%(27 / 75)$ & 0.68 \\
3 months & $69 \%(9 / 13)$ & $55 \%(46 / 83)$ & $46 \%(35 / 76)$ & 0.22 \\
6 months & $85 \%(11 / 13)$ & $69 \%(56 / 81)$ & $54 \%(40 / 74)$ & 0.05 \\
12 months & $86 \%(12 / 14)$ & $77 \%(61 / 79)$ & $61 \%(43 / 71)$ & 0.01 \\
Unilateral NS & & & & \\
1 month & $43 \%(3 / 7)$ & $39 \%(11 / 28)$ & $31 \%(11 / 36)$ & 0.31 \\
3 months & $50 \%(3 / 6)$ & $48 \%(13 / 27)$ & $37 \%(13 / 35)$ & 0.17 \\
6 months & $66 \%(4 / 6)$ & $58 \%(15 / 26)$ & $40 \%(14 / 35)$ & 0.10 \\
12 months & $71 \%(5 / 7)$ & $64 \%(16 / 25)$ & & \\
\hline
\end{tabular}

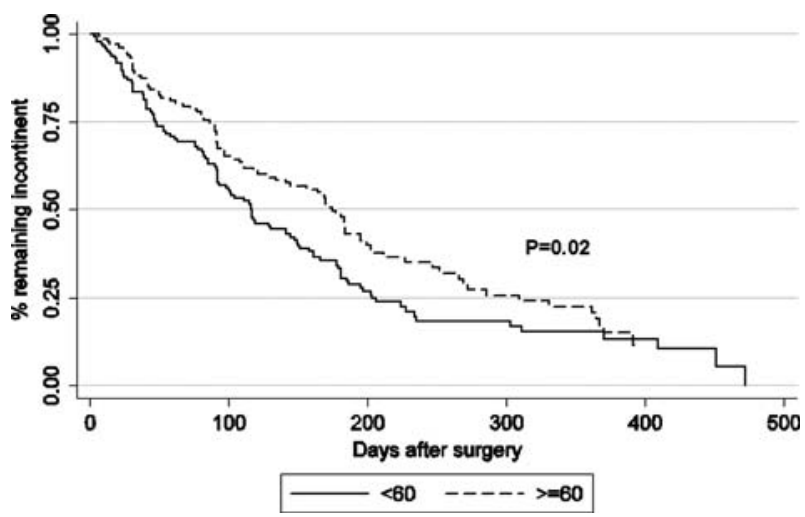

Fig. 1 Kaplan-Meier estimates for continence recovery following robotic-assisted laparoscopic radical prostatectomy (RLRP). The distribution is significantly different between the two age groups with the younger group taking shorter time to recover $(P=0.02)$

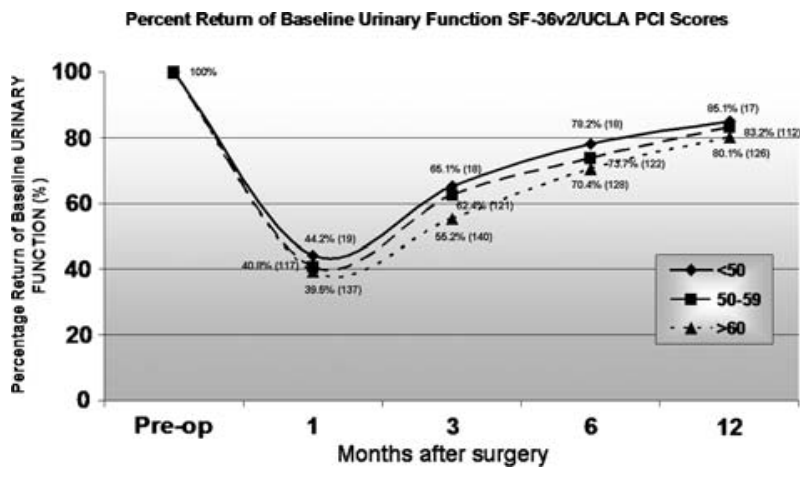

Fig. 2 Age-stratified, mean percent return of baseline (pROB) urinary function based on RAND-36 Item Health Survey v2/ UCLA Prostate Cancer Index (SF-36/UCLA PCI) scores
HRQOL questionnaire urinary function results revealed 1, 3, 6 and 12 month pROB urinary function of $44.2,65.1,78.2$ and $85.1 \%$ for men aged $<50 ; 40.8,62.4$, 73.7 and $83.2 \%$ for men aged $50-60 ; 39.5,55.2,70.4$ and $80.1 \%$, respectively. No significant differences between the age groups were observed at any of the follow up time-points (Fig. 2).

\section{Potency outcomes}

The youngest men ( $<50$ years old) had significantly higher mean pre-operative SF-36 questionnaire sexual function scores $(P=0.01)$. Similarly, with regards to SHIM scores, $95.2,88.4$ and $78.7 \%$ of men aged $<50$, $50-60,>60$, respectively, were potent pre-operatively $(P=0.04)$. Potency outcomes of the age-stratified groups are summarized in Table 2. Among men with bilateral nerve preservation, younger men had a superior potency recovery when compared to older men, with the trend achieving statistical significance at 6 and 12 months $(P=0.05$ and 0.01 , respectively). The youngest men $(<50)$ had an earlier return to strict potency (mean of 87 days), compared with the other age groups (50-59 and $\geq 60$ years old, mean of 109 and 105 days, respectively) $(P=0.01)$.

Kaplan-Meier curve analysis demonstrated a significantly improved return to potency for men $<60$ years when compared to men aged $\geq 60(P<0.01)$ (Fig. 3). HRQOL questionnaire sexual function results, for men with bilateral nerve preservation, revealed 1, 3, 6 and 12 month pROB sexual function of $67.8,71.1,76.7$ and $90.2 \%$ for men aged $<50 ; 57.3,66.9$, 72.4 and $78 \%$ for men aged 50-60; 50.4, 54.7, 60.6 and $66.4 \%$ for men $>60$, respectively. Although there was a 


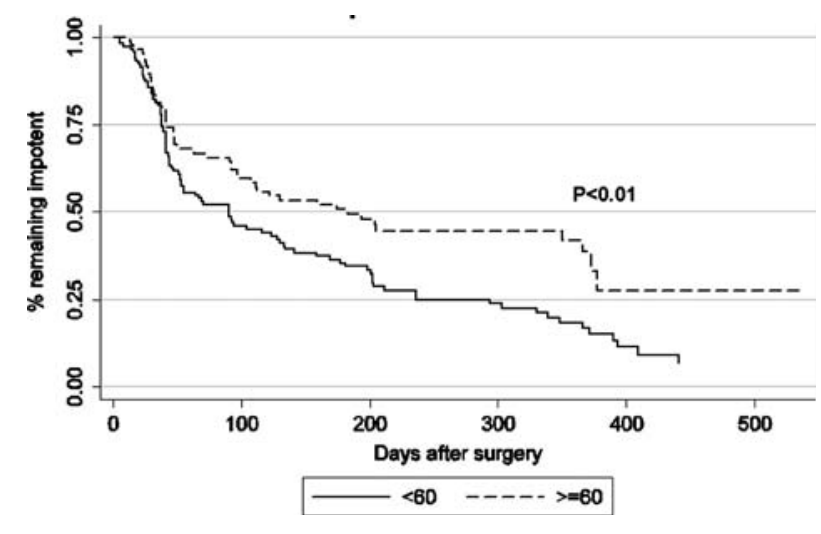

Fig. 3 Kaplan-Meier estimates for potency recovery following RLRP. Time to potency recovery distribution is significantly different between the two age groups $(P=0.005)$

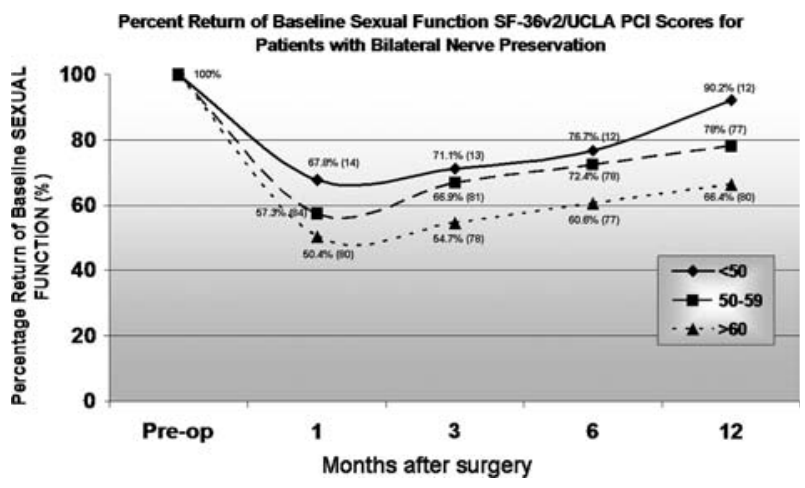

Fig. 4 Age-stratified, mean pROB sexual function for men with bilateral nerve preservation based on SF-36/UCLA PCI scores

noticeable trend, pROB sexual function did not reach statistical difference between age groups at all followup intervals (Fig. 4).

\section{Discussion}

The introduction of robotic-assistance has the potential to improve surgical outcomes and reduce the steep learning curve associated with conventional LRP. The three-dimensional visualization of periprostatic anatomy and the unique, wristed instrumentation afford the surgeon incredible precision for meticulous tissue dissection. Improved surgical outcomes, such as minimal blood loss [19] and reduced patient morbidity [7], have been well described with laparoscopic surgery. Herrell and Smith [20] noted that the optimal oncologic, continence and potency outcomes of RRP have reached a plateau, and RLRP may allow urologists to further improve surgical technique and provide superior results. Many consider that robotic-assistance increases the potential for better NVB preservation [21].
Presently, any claims of superior outcomes related to erectile function for robotic or open prostatectomy cannot be justified [22]. With a lack of randomized studies and the lack of consensus regarding the optimal instrument for assessing erectile dysfunction, such a claim is difficult to validate.

From an oncological perspective, the finding of a PSM after radical prostatectomy is an independent predictor of biochemical recurrence and represents a proxy for local disease control. Several experienced robotic centers have reported favorable short-term oncologic control, with PSM rates ranging from 4.5 to $16 \%[5-7,11,20,23]$ for pathologically organ-confined (pT2) disease. In most RLRP series, PSM rates decrease as greater familiarity with the procedure is obtained. Recent open series show PSM rates from 2.9 to $28 \%$ for pT2 disease [2, 3, 22, 24-26]. In our series, we have demonstrated an overall pT2 PSM of $14.7 \%$ (37/251). There was also significant higher pT2 PSM in younger men when compared to those $>60$ years old $(P=0.03)$. We attribute this finding to the significant prostate size difference between the age groups, as demonstrated in Table 1 . In RRP and LRP series, prostate size has been shown to be inversely related to PSMs [27-30]. Similarly, we have also demonstrated the higher incidence of pT2-PSM in men with smaller prostate volumes [31]. Other than smaller prostate sizes, younger men also had a higher degree of interfascial nerve sparing (unilateral or bilateral) when compared to the older groups. Interfascial nerve preservation has been associated with higher postero-lateral PSM rates, particularly for pT2-disease related to inadvertent capsulotomy $[11,31]$.

When comparing functional outcomes among other published series, it is important to consider other patient cohort characteristics that may influence such results, particularly age. Patient age is a well defined, independent risk factor of erectile dysfunction. Similarly, patient age has also been associated with declines in lean body mass and the quantitative and functional character of skeletal muscle [32]. Equally important, particularly for the acquirement of pelvic control for urinary continence, is the age-related decline of neuronal plasticity [33]. Aging humans show significant impairment in acquiring new tasks; reduced neuronal excitability in the hippocampal neurons is hypothesized to play a role in this phenomenon. As such, older men may take longer to acquire the necessary pelvic floor reflex in order to achieve complete urinary continence.

Using a strict criterion of zero pads per day with occasional pad use for security reasons, several 
academic institutions have reported continence rates of $80-95 \%[5,7,9,34]$ at 12 months after surgery. Such results are comparable with overall subjective continence outcomes of $84 \%$ in this series. After stratifying results by age, younger men were noted to achieve subjective continence earlier $(67 \%$ for men $<50$ vs $41 \%$ for men $>60$, at 3 months; $P=$ $0.03)$. Similar finding were observed in the KaplanMeier curve estimates (Fig. 1). However, at 12 months post-operatively, men of all age groups were noted to have similar continence rates. RLRP results in this study differ from previously published data in open RRP series [1,3] where better longterm continence outcomes were observed in younger men. Improved continence outcomes in older patients may be attributed to better apical dissection, especially in larger prostate sizes, provided by the superior visualization and finer dissection of the surgical planes with robot assistance. As mentioned above, age-related decline in neuronal excitability and synaptic recruitment, coupled with decreased muscle function may help explain the early differences observed between the age groups. In this series, with increased time, older patients appear to catch up and acquire the necessary neuro-motor function to achieve pad-free continence.

Mean pROB urinary function was not statistically significant between age groups for all time intervals. At 12 months post-operatively, a mean percentage of baseline, pre-operative urinary function score (which is not equivalent to urinary continence) of $85.1,83.2$ and $80.1 \%$ of men aged $<50,50-59$ and $>60$ years old, respectively, was observed. Overall pROB as a group compared favorably to the results of other contemporary radical prostatectomy series $(64-74 \%)$ using similar validated questionnaires [8, 34, 35].

Moreover, independent factors associated with recovery of erections after surgery include younger patient age, stronger preoperative erections, preservation of NVBs, surgical technique and surgeon experience [23, 36, 37]. Menon et al. [38] previously presented their technique for lateral prostatic fascia preservation during RLRP and have demonstrated outstanding sexual function outcomes when compared to other contemporary series. Their recently published data comparing their own series of lateral prostatic fascia sparing and conventional nerve sparing RLRP resulted in significantly superior potency outcomes in the former group $(97 \%$ vs $74 \%$, $P=0.002$ ) [39].

In this study, preoperative potency (defined as a SHIM score $\geq 20$ ) was significantly different among the age groups $(P=0.04)$. When comparing different age group functional outcomes, men below 50 years old were noted to have an earlier return to subjective potency, which was statistically significant at 3 and 6 months follow-up. Twelve-month potency rates for men with bilateral nerve preservation in each age group ( $<50,50-59$, and $\geq 60$ years old) were 86,77 and $61 \%$, respectively $(P=0.01)$. These results are comparable to outcomes reported by expert, open surgeons such as Walsh [25] (91, 75 and 58\%, respectively) and Catalona [26] (90, 80 and 60\%, respectively). A trend towards better long-term subjective results in younger men when compared with older age groups with either bilateral or unilateral nerve preservation is also demonstrated in the Kaplan-Meier analysis (Fig. 3).

Other published data using similar validated questionnaires in assessing sexual function at 12 months post-LRP reported inferior results (39-64\%) compared to these results of $90.2,78$ and $66.4 \%$ in the $<50,50-59$, and $\geq 60$ year old age groups, respectively [34, 35, 40]. The pROB results showing no significant difference between age groups at all time-points may have been affected by our mean preoperative sexual function scores, which showed significant differences between age groups $(P=0.01)$. The higher preoperative function paralleled by higher expectations of recovery postoperatively in younger men may explain the difficult for these patients to achieve a greater percent of baseline sexual function by SF-36 v2/UCLA PCI subscale analysis compared to older men.

Overall long term sexual function outcome data in younger men is similar to other published RRP data [37]. Differences in reporting potency data, however, has made it difficult to compare data with other published series. Presenting both subjective clinical and questionnaire assessment of functional outcomes has allowed us to present a more realistic view of the actual HRQOL outcomes of patients after RLRP.

This study has several limitations that warrant discussion. The retrospective nature of the study, based on data from a single institution must be pointed out. Similarly, our study is also limited by its sample size. Questionnaire response and clinical follow-up was not complete for all patients. Many of the patients were from outside our geographic area and were followed locally. Along with clinical visits, telephone interviews and mailed HRQOL questionnaires allowed for an $85 \%$ completion rate $(255 / 300)$.

RLRP experience has increased in the past few years from 247 cases performed in 2001 [7] compared to a predicted 35,000 cases in 2006. At the time of writing, we have performed over 900 RLRP cases and our surgical margin rates have dramatically improved 
since our initial case in 2003. The frequently debated issue regarding which surgical approach is best for the management of clinically localized pCA [26-28] will ultimately depend on the surgeon's experience in carrying out these procedures. While we patiently await long-term RLRP data to provide evidence for equivalent cancer control to RRP data, our study reaffirms that RLRP definitely provides favorable quality of life outcomes for all age ranges studied.

\section{Conclusion}

Our data suggests that, as is seen using retropubic and perineal approaches, younger men who undergo nervesparing RLRP will likely have an earlier return of continence and potency compared to older men. However, continence outcomes appear to be equal among age groups after 1 year of follow-up. Younger men continue to report superior potency outcomes compared to older men over the first postoperative year. Such findings are valuable in counseling patients undergoing this procedure.

\section{References}

1. Eastham JA, Kattan MW, Rogers E et al (1996) Risk factors for urinary incontinence after radical prostatectomy. J Urol 156:1707-1713

2. Noh C, Kshirshagar A, Mohler JL (2003) Outcomes after radical retropubic prostatectomy. Urology 61:412-416

3. Kundu SD, Roehl KA, Eggener SE et al (2004) Potency, continence and complications in 3,477 consecutive radical retropubic prostatectomies. J Urol 172:2227-2231

4. Twiss C, Slova D, Lepor H (2005) Outcomes of men younger than 50 years undergoing radical prostatectomy. Urology 66:141-146

5. Menon M, Shrivastava A, Sarle R et al (2003) Vattikuti Institute Prostatectomy: a single-team experience of 100 cases. J Endourol 17:785-790

6. Smith JA, Herrell SD (2005) Robotic-assisted laparoscopic prostatectomy: do minimally invasive approaches offer significant advantages? J Clin Oncol 23:8170-8175

7. Ahlering TE (2006) News and topics. Robotic prostatectomy: is it the future? Urol Oncol Semin Orig Investig 24:1-3

8. Link RE, Su LM, Sullivan W et al (2005) Health related quality of life before and after laparoscopic radical prostatectomy. J Urol 173:175-179

9. Rassweiler J, Hruza M, Teber D et al (2006) Laparoscopic and robotic assisted radical prostatectomy-critical analysis of results. Eur Urol 49:612-624

10. Chien GW, Mikhail AA, Orvieto MA et al (2005a) Modified clipless antegrade nerve preservation in robotic-assisted laparoscopic radical prostatectomy with validated sexual function evaluation. Urology 66:419-423

11. Zorn KC, Gofrit ON, Orvieto MA et al (2006) Roboticassisted laparoscopic prostatectomy: functional and pathologic outcomes with interfascial nerve preservation. Eur Urol (in press) DOI: 10.1016/j.eururo.2006.10.019
12. Shalhav AL, Orvieto MA, Chien GW et al (2006) Minimizing knot tying during reconstructive laparoscopic urology. Urology 68:508-513

13. Chien GW, Mikhail AA, Orvieto MA et al (2005b) Modified clipless antegrade nerve preservation in robotic-assisted laparoscopic radical prostatectomy with validated sexual function evaluation. Urology 66:419-422

14. Van Vethoven RF, Ahlering TE, Peltier A et al (2003) Technique for laparoscopic running urethrovesical anastamosis: the single knot method. Urology 61:699-702

15. Orvieto MA, Chien GW, Laven B et al (2004) Eliminating knot tying during warm ischemia time for laparoscopic partial nephrectomy. J Urol 172:2292-2295

16. Alsikafi NF, Brendler CB (1998) Surgical modifications of radical retropubic prostatectomy to decrease incidence of positive surgical margins. J Urol 159:1281-1285

17. Litwin MS, McGuigan K (1999) Accuracy of recall in healthrelated quality of life assessment among men treated for prostate cancer. J Clin Oncol 17:2882-2888

18. Krupski TL, Saigal CS, Litwin MS (2003) Variation in continence and potency by definition. J Urol 170:1291-1294

19. Farnham SB, Webster TM et al (2006) Intraoperative blood loss and transfusion requirements for roboric-assisted radical prostatectomy versus radical retropubic prostatectomy. Urology 67:360-363

20. Herrell SD, Smith JA (2005) Laparoscopic and robotic radical prostatectomy: what are the real advantages? BJU Int 95:3-9

21. Joseph JV, Vicente I, Madeb R et al (2005) Robot-assisted vs pure laparoscopic radical prostatectomy: are there any differences? BJU Int 96:39-42

22. Lepor H (2006) Open versus robotic radical prostatectomy. Urol Oncol Semin Orig Investig 24:91-93

23. El-Hakim A, Leung RA, Tewari A (2006) Robotic prostatectomy: a pooled analysis of published literature. Expert Rev Anticancer Ther 6:11-20

24. Bianco FJ, Scardino PT, Eastham JA (2005) Radical prostatectomy: long term cancer control and recovery of sexual and urinary function ("Trifecta"). Urology 66:83-94

25. Walsh PC, Partin AW, Epstein JI (1994) Cancer control and quality of life following anatomical radical retropubic prostatectomy: results at 10 years. J Urol 152:1831-1836

26. Catalona WJ, Carvajal GF, Marger DE et al (1999) Potency, continence and complication rates in 1870 consecutive radical retropubic prostatectomies. J Urol 162:433-438

27. D'Amico AV, Whittington R, Malkowicz SB et al (1998) A prostate gland volume of more than $75 \mathrm{~cm}^{3}$ predicts for a favorable outcome after radical prostatectomy for localized prostate cancer. Urology 52:631-636

28. Hsu EI, Hong EK, Lepor H (2003) Influence of body weight and prostate volume on intraoperative, perioperative, and postoperative outcomes after radical retropubic prostatectomy. Urology 61:601-606

29. Freeland SJ, Isaacs WB, Platz EA et al (2005) Prostate size and risk of high-grade, advanced prostate cancer and biochemical progression after radical prostatectomy: a Search database study. J Clin Oncol 23:7546-7554

30. Chang CM, Moon D, Gianduzzo TR et al (2005) The impact of prostate size in laparoscopic radical prostatectomy. Eur Urol 48:285-290

31. Zorn KC, Orvieto MA, Mikhail AA et al (2007) Effect of prostate weight on operative and postoperative outcomes of robotic assisted laparoscopic prostatectomy. Urology (in press)

32. Proctor DN, Melton LJ III, Khosla S et al (2000) Relative influence of physical activity, muscle mass and strength on bone density. Osteoporos Int 11:944-952 
33. Disterhoft JF, Oh MM (2006) Learning, aging and intrinsic neuronal plasticity. Trends Neurosci 29:587-599

34. Soderdahl DW, Davis JW, Schellhammer PF et al (2005) Prospective longitudinal comparative study of health-related quality of life in patients undergoing invasive treatment for localized prostate cancer. J Endourol 19:318-326

35. Yang BK, Young MD, Calingaert B et al (2004) Prospective and longitudinal patient self-assessed health related quality of life following radical perineal prostatectomy. J Urol 172:264-268

36. Saranchuck JW, Kattan MW, Elkin E et al (2005) Achieving optimal outcomes after radical prostatectomy. J Clin Oncol 23:4146-4151
37. Rabbani F, Stapleton AM, Kattan MW et al (2000) Factors predicting recovery of erections after radical prostatectomy. J Urol 164:1929-1934

38. Kaul S, Bhandari A, Hemal A et al (2005) Robotic radical prostatectomy with preservation of the prostatic fascia: a feasibility study. Urology 66:1261-1265

39. Menon M, Kaul S, Bhandari A et al (2005) Potency following robotic radical prostatectomy: a questionnaire based analysis of outcomes after conventional nerve sparing and prostatic fascia sparing techniques. J Urol 174:2291-2296

40. Rogers CG, Su LM, Link RE et al (2006) Age stratified functional outcomes after laparoscopic radical prostatectomy. J Urol 176:2448-2452 